\title{
Lessons from the management of acute myocardial infarction
}

\section{Pearson}

Heart 2005;91(Suppl II):ii28-ii30. doi: 10.1136/hrt.2005.062075

The National Service Framework for coronary heart disease set a number of challenging targets for the care of patients following an acute myocardial infarction. The Myocardial Infarction National Audit Project (MINAP) was devised to monitor progress and has been notably successful in winning professional support and participation and helping trusts to meet these targets. The new challenge is in translating this success to other areas of medicine. Heart failure is one such area, although it poses a number of difficulties relating primarily to disease definition and the definition of a successful outcome. MINAP was overseen by a multidisciplinary group of stakeholders, including patient organisations, and was project managed by a professionally led team at the Royal College of Physicians. Successful projects must retain confidence of all stakeholders and in part this depends on ensuring that timelines are met. Central monitoring of returns and anticipation of problems has been an important component of data completeness and quality. Next day updates to those collecting the data and more detailed quarterly reports for clinicians and chief executives within days of quarter end have been vital. Change depends on clinicians and managers working together. But most importantly, the attention to detail outlined above means the data have been believed and the resulting change for patients has been remarkable.

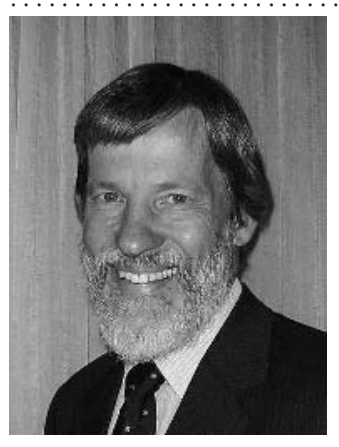

Correspondence to: Professor Mike Pearson, Clinical Effectiveness and Evaluation Unit, Royal College of Physicians, 11 St Andrews Place, Regent's Park, London NW1 4LE, UK; michael.pearson@ rcplondon.ac.uk $\mathrm{n}$ recent years, the Clinical Effectiveness and Evaluation Unit at the Royal College of Physicians (RCP) has developed expertise in the measurement of how the National Health Service provides care for patients suffering from various diseases. The most recent and notable project has been the Myocardial Infarction National Audit Project (MINAP), established as a response by the profession to the audit requirements of the National Service Framework (NSF) for coronary heart disease, published in 2000. ${ }^{1}$

Perhaps the unfortunate message from all of the audits that the RCP has conducted is that in are much more likely to perform appropriately if they know someone is watching and recording what they do. Furthermore, this is as true in large teaching hospitals as it is in small hospitals. health care, as in everyday life activities, people
The experience of measuring health care interventions has taught the RCP four simple lessons:

- define the denominator

- keep it simple

- make it happen

- sell the story.

\section{SETTING THE PARAMETERS}

Any form of medical audit has to overcome many different hurdles. It is necessary to define what constitutes success for an intervention and to ensure that the patient is followed up in order to determine whether or not the intervention has been successful. Ideally, the measure must be simple and must be readily recordable in busy clinical practice. This requires only collecting details, such as case mix variables, that are essential for interpretation. Physicians prefer to be treating patients rather than recording data, and their motivation to do so is reduced by beliefs that they already know what is best for their patient.

Defining success in heart failure poses a particular difficulty. The Royal College of Physicians worked with the National Institute for Clinical Excellence (NICE) to develop its guideline on the management of chronic heart failure. ${ }^{2}$ Despite this excellent document, it remains unclear whether a successful outcome with respect to heart failure care is one that is:

- keeping more patients alive

- keeping patients out of hospital

- making patients feel better

- helping patients to do more.

MINAP benefited from being able to measure success against the targets for the care of patients following an acute myocardial infarction set out in the NSF. These included:

- $75 \%$ of eligible patients should be thrombolysed within 30 minutes of arrival at hospital by April 2002

- by April 2002, 90\% of patients should be discharged from hospital following a heart attack having been prescribed secondary prevention drugs.

Abbreviations: COPD, chronic obstructive pulmonary disease; MINAP, Myocardial Infarction National Audit Project; NICE, National Institute for Clinical Excellence; NSF, National Service Framework; RCP, Royal College of Physicians; QALY, quality adjusted life-year 
MINAP benefited too from a clear definition based on ECG and enzyme values that are widely accepted and understood. The door-to-needle target may be a simple process measure that is a proxy that reflects the urgency and efficiency with which health professionals organise their systems of care.

In contrast, the NICE guideline defines heart failure as:

\begin{abstract}
...a complex syndrome that can result from any structural or functional cardiac disorder that impairs the ability of the heart to function as a pump to support a physiological circulation. The syndrome of heart failure is characterised by symptoms such as breathlessness and fatigue, and signs such as fluid retention.
\end{abstract}

The NICE guideline also says:

There is no single diagnostic test for heart failure, and diagnosis relies on clinical judgement based on a combination of history, physical examination and appropriate investigations.

The NICE definition does not allow for an easy measure for audit purposes. If only hospital admissions were to be studied, then it would be possible to work on the basis of a discharge diagnosis made by a clinical team. This worked for the RCP's chronic obstructive pulmonary disease (COPD) audit. If a patient went home with a discharge diagnosis of COPD, it is not unreasonable to expect that they ought then to have been treated in accordance with the COPD management guidelines.

But while such a definition might hold true for heart failure patients admitted to secondary care, a different approach is needed in primary care. Heart failure in primary care might be defined on the basis of:

- drugs received

- referral for echocardiography

- a secondary care diagnosis

- any recorded heart failure label.

Clearly a single definition is needed, one that can be applied anywhere. At present, the heart failure community is a long way short of agreeing such a definition.

There is value of starting with an audit in the hospital sector. The RCP's audit of COPD in hospital patients ${ }^{3}$ showed huge variance between the best and the worst hospitals, even for straightforward variables such as sputum recording (needed to guide antibiotic choice) or the presence of leg oedema (an important prognostic feature). Some hospitals, including major teaching hospitals, made no record of these on any case. The COPD study included also a clear cut outcome-death within three months with an acute admission of COPD - and showed that even after controlling for case mix (which required a great deal of attention) small hospitals had a $50 \%$ greater mortality. ${ }^{4}$ It is quite possible that similar variance will apply to heart failure.

\section{RECORDING THE DATA}

A recording system is a fundamental requirement of any audit. MINAP is based on a user friendly electronic data recording system that uses secure and encrypted transmission of data via the internet or NHS Net to link with a central system. Patient records are analysed centrally and comparisons of local with national aggregate performance are made available to participating hospitals on-line. The system meets all the requirements of data protection legislation and confidentiality. In addition, it is linked to the Office for National Statistics allowing for provision of case fatality data.
All hospitals in England and Wales are now entering data to MINAP. Importantly, the electronic system provides prompt feedback reports to the user hospitals, usually within 24 hours. Any audit system needs to feedback data as near as possible to real time rather than reporting six or 12 months later when many of the junior staff and nurses will have moved on.

MINAP began with a very limited dataset, recording only important and relevant information. However, having established successful collection, it has been possible to slowly expand the dataset to enable links to the ambulances and angioplasty services.

The audit results should only be used to change practice if they are demonstrably both valid and reliable. Past experience with stroke audit had shown that this cannot be presumed. The Intercollegiate Stroke Audit working party included all the important stakeholders, including patients, and set out the audit standards. These were then submitted to a formal consensus (modified Delphi) survey before the audit questions were developed and piloted for validity and reliability. ${ }^{5}$ Despite this preparation, initial analysis of this first version yielded results that were little better than chance. The tool was revised and a detailed help booklet for those collecting the data was developed. In each subsequent study the inter-rater reliability has been checked and has remained very good. Overall, the RCP experience showed the need to:

- set a few, clear, simple targets

- collect only what is needed and not what it might be nice to know

- ensure that data are clearly defined so they mean the same to clinicians, audit staff, and managers

- check data are available, collectable, and robust

- ensure units understand what is being asked of them.

\section{THE MINAP EXPERIENCE}

MINAP was a huge undertaking and took a long time to implement. Recruitment began in October 2000 with a few pilot sites, took 18 months to get the first $90 \%$ of sites, and then a further 18 months to bring in the last $10 \%$. It now has cumulative data on more than 300000 cases within the system.

Next day feedback given to hospitals informs them where they are against both national targets and the rest of the country. The data have been used to change practice. When MINAP started, about $38 \%$ of patients were getting thrombolysis within 30 minutes of arrival in hospital. This had risen to $79 \%$ by October 2003 and $84 \%$ for the third quarter of 2004 , showing that the improvements are being sustained. In the midst of a very busy acute admission process, this is an unprecedented response. Furthermore, this is not a selective group of patients or hospitals, but represents an improvement across the whole of England and Wales.

Initially, data collected through MINAP were only to be fed back to the participating hospitals. However, as the data have matured, quarterly reports are sent to the strategic health authorities and to the Department of Health. In addition, there is now an annual report to the public: increasingly, data need to be shared with the public as it is the public that ultimately determines expenditure on health care. What began as a medical audit has evolved into a system for monitoring clinical performance. Moreover it is reporting not just to the health service "policeman" but also to the ultimate users, the public.

Provisional analysis of (unadjusted) 35 day all cause mortality data on patients with an admission diagnosis of myocardial infarction having thrombolytic treatment, from 
October 2000 to March 2003, suggests that there has been a fall in mortality of the same order as predicted from the clinical trials. While it is too early to say whether or not this is necessarily due to MINAP, it is certainly encouraging.

Over the same period there have also been changes in the institution of secondary prevention measures, with substantial increases in the proportion of patients going home following a myocardial infarction with angiotensin converting enzyme (ACE) inhibitors, $\beta$ blockers, statins, and aspirin. This again represents a huge change in behaviour of staff within hospitals.

Linking to the Office for National Statistics means that for the $90 \%$ or so of patients with a recorded NHS number, it is possible to track survival. The data show that for patients who are alive at 60 days after their myocardial infarction, mortality in the following year is $2 \%$ for those who are taking a statin, but is $4.5 \%$ for those who are not taking a statin. Although these data are not adjusted for potential confounding factors, they do suggest that improved prescribing behaviour within hospitals is having a beneficial effect.

\section{COSTS OF AUDIT}

MINAP has demonstrated reduced deaths at one month and at one year over the period of data collection. The RCP's stroke audit has shown improvements over six years, although of a lesser magnitude than MINAP in four years. These are most encouraging although the causal relation to these benefits is unproven, but if they are accepted it is possible to make some crude calculations of cost benefit. If we make a conservative estimate of the total number of cases and the percentage fall in mortality and assume that just half of this has been due to MINAP, we can make a few "back of the envelope" calculations. The cost for collecting data per case is about $£ 15$ in MINAP, while an in-patient admission costs around $£ 1500$ depending on the length of stay in hospital. From the change in observed mortality, MINAP appears to be associated with a cost per quality adjusted lifeyear (QALY) in the range of $£ 100-350$. For most pharmaceutical products a cost per QALY of a $£ 1000-1200$ or even $£ 5000$ is deemed acceptable, while NICE works on a cost per QALY threshold of $£ 20$ 000-30 000. A similar calculation related to the stroke data produce a figure of under $£ 100$ per QALY. Therefore, even if this rough calculation is an order of magnitude out, getting care better organised by measuring seems to offer very good value for money.

\section{KEY FEATURES TO MAKE IT HAPPEN}

MINAP has been a team effort. An efficient central team and effective IT solutions are of no value if the colleagues in trusts and health authorities elect not to participate. Many different groups of people have to be kept on side, and each has different interest in the results. They include:

- Clinical staff-need frequent detailed reports to promote team working/local efficiency

- Chief executives-need quarterly reports for clinical governance/appraisal purposes and also for resource allocation

- Regions and Department of Health-need annual reports to ensure that targets have been met

- The public and press - should receive annual reports but it is unclear which form is most appropriate.

Overall, the MINAP experience has suggested that an unprecedented rate of change can be achieved but that this needs central coordination. This comprises:
Learning points

- The Myocardial Infarction National Audit Project (MINAP) has helped acute NHS trusts to meet the targets on acute myocardial infarction treatment set in the National Service Framework for coronary heart disease, with clear patient benefit

- The success of MINAP can be attributed to the professional support achieved for the project. This, in turn, relates to the care taken in design and operation of the audit and the provision of prompt feedback to clinicians and managers

- Similar methodology could be used to improve the provision of care for patients with post-myocardial infarction heart failure

- a steering group that includes all stakeholders

- a robust database managed by those who understand the data (Central Cardiac Audit Database) that is trusted to provide clinical confidentiality "guarantees" for patients, for clinicians, and for hospitals

- a central team to help and support the staff in hospitals, to constantly monitor data quality, and to develop and communicate reports relevant to each audience

\section{CONCLUSION}

The MINAP experience shows a potential approach that could be applied to heart failure. Defining measurable diagnostic and success criteria have to be overcome but it would be possible to mimic the "COPD approach" in the first instance for patients admitted to hospital. Bridging the gap to measure in both primary and secondary care will be a greater challenge. The audit experiences of MINAP, stroke, and COPD could be applied to heart failure. Some "pump priming" money to get started on defining and agreeing some universal definitions would be helpful. Thereafter the NHS ought to fund such work as this does seem to be a cost effective means of improving clinical practice.

Changing practice through audit is a huge challenge but is worth considering. This is not a new concept. In 1917, in his seminal work $A$ study in hospital efficiency, the US surgeon Dr Ernest A Codman, one of the founders of what we now call outcomes management, wrote: "Every hospital should follow every patient it treats long enough to determine whether or not the treatment was successful and to inquire 'if not, why not?' with a view to preventing similar failures in future". Clinicians around the world have been remarkably slow to catch on.

\section{REFERENCES}

1 Department of Health. National service framework for coronary heart disease. London: Department of Health, 2000.

2 National Institute for Clinical Excellence. Management of chronic heart failure in adults in primary and secondary care. Clinical Guideline 5; 2003.

3 Roberts CM, Ryland I, Lowe D, et al. Audit of acute admissions of COPD: standards of care and management in the hospital setting. Eur Respir J 2001;17:343-9.

4 Roberts CM, Barnes S, Lowe D, et al. Evidence for a link between mortality in acute COPD and hospital type and resources. Thorax 2003;58:947-9.

5 Gompertz PH, Irwin P, Morris R, et al. Reliability and validity of the intercollegiate stroke audit package. J Eval Clin Pract 2001;7:1-11. 